\title{
Oversized post-dilatation of current bioresorbable vascular scaffolds: kill or cure?
}

Published on 18 March 2016

Clemens von Birgelen ${ }^{1,2 *}, \mathrm{MD}, \mathrm{PhD}$; Mounir W.Z. Basalus ${ }^{1}, \mathrm{MD}, \mathrm{PhD}$

1. Department of Cardiology, Thoraxcentrum Twente, Medisch Spectrum Twente, Enschede, The Netherlands; 2. Health Technology and Services Research, MIRA - Institute for Biomedical Technology and Technical Medicine, University of Twente, Enschede, The Netherlands

Bioresorbable scaffolds, of which most experience has been obtained with the poly-L-lactide (PLLA)-based Absorb bioresorbable vascular scaffold (BVS; Abbott Vascular, Santa Clara, CA, USA), have the potential to overcome the limitations of metallic drug-eluting stents (DES), as they temporarily scaffold the diseased coronary vessel and then disappear. After degradation of the BVS, the coronary artery can restore its vasomotion and it may show favourable vascular remodelling ${ }^{1,2,3}$. During the last four years, more than 100,000 patients have been treated with BVS, while several million patients have been treated with metallic DES which showed favourable clinical outcomes in randomised clinical trials, including low target vessel revascularisation and stent thrombosis rates 4,5

So why has there not been a surge in the use of BVS in our daily clinical practice? A simple but important reason is the efficacy and proven long-term safety of the current-generation DES. When we recall the introduction of bare metal stents and - more than a decade later - DES, there were compelling reasons to introduce these devices rapidly, as in both cases the new devices solved or mitigated major clinical problems, for which there was no similarly effective alternative. BVS promise to overcome the problem of neoatherosclerosis formation in DES, which has been observed in both early and newergeneration $\mathrm{DES}^{6}$; however, considering the relatively low long-term event rates of patients treated with modern DES, this problem seems to be not overly common. The benefits of BVS are presumed to pay off in lower event rates after several years, but this remains to be demonstrated in ongoing large trials. While patients of all ages intuitively embrace the concept of a dissolving device, one may expect to see the greatest potential clinical benefit in the young and middle-aged.

In addition, recent clinical studies and meta-analyses have shown some signals of a potential increase in risk of (early) stent thrombosis with bioresorbable scaffolds $7,8,9$. While the significance of this finding is still unclear, the timing of scaffold thrombosis, early after implantation, suggests a relation with the relatively thick strut dimensions, the apposition to the vessel wall, and/or implantation techniques (e.g., aggressiveness of lesion preparation, adequate device sizing with or without intracoronary imaging, employment of post-dilatation, balloon size and pressures used). For thick-strut coronary stents and scaffolds alike, it is of paramount importance to appose the device well to the vessel wall ${ }^{9}$. In clinical practice, there is frequently a need for stent postdilatation and overexpansion to achieve full device apposition ${ }^{10}$, and the same holds true for BVS. Severely oversized post-dilatations of metallic stents are generally well tolerated without destructive consequences to the metal frame, but may have a significant effect on stent geometry ${ }^{10}$. The consequences of BVS overexpansion have not been fully examined, so it was high time that a thorough evaluation of this matter was performed and published.

In the current issue of Eurolntervention, Foin et al report the results of meticulous bench-top experiments on the overexpansion of BVS with non-compliant balloons of increasing diameters ${ }^{11}$. While BVS of different sizes could be oversized by $1 \mathrm{~mm}$ in a non-constrained setting, only overexpansion with a noncompliant balloon $0.5 \mathrm{~mm}$ larger than the BVS size was feasible in a constraining eccentric arterial lesion model. Excessive overexpansion of BVS beyond that limit resulted in scaffold fractures and a decrease in mechanical support, as impressively demonstrated by mechanical point force measurements. This loss of mechanical support may trigger adverse clinical events. The work of Foin et al confirms the "no more than 0.5 mm larger" instruction for BVS postdilatation with non-compliant balloons which was provided by the device manufacturer. In addition, interesting insights with 2D and 3D optical coherence tomography (OCT) in fractured BVS samples demonstrated an advantage of 3D OCT in detecting fracture sites. Partial overexpansion of BVS with noncompliant balloons beyond the safety limit of $0.5 \mathrm{~mm}$ resulted in fractures which were located, in particular, in the region between the post-dilated and nonpost-dilated segments ${ }^{11}$. In parallel with these findings, previous studies of metallic DES demonstrated the greatest stent deformation to be present just in that region (Figure 1) ${ }^{10}$
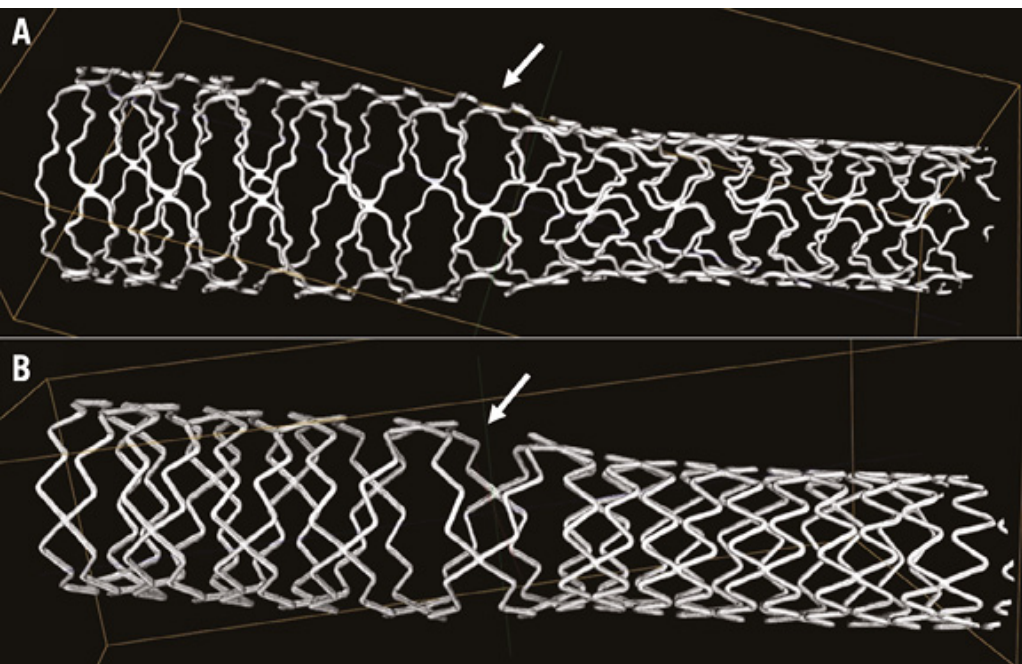

Figure 1. Micro-computed tomographic assessment following extremely oversized partial post-dilatation of metallic DES with an open-cell design. The maximum stent deformation with the largest cells (arrowheads) was seen in the transitional region between the $1.5 \mathrm{~mm}$ overexpanded and the non-post-dilated segments (A: TAXUS Liberté ${ }^{\mathrm{TM}}$; Boston Scientific, Natick, MA, USA; B: Resolute ${ }^{\mathrm{TM}}$; Medtronic, Santa Rosa, CA, USA). The region of maximum stent deformation corresponds with the scaffold region in the study by Foin et al ${ }^{11}$ which showed many BVS fractures after excessive partial overexpansion. Quantitative data on DES deformation have previously been reported ${ }^{10}$.

The safety limit of BVS overexpansion emphasises the fact that an optimised sizing of BVS is of paramount importance. In a way, this is an echo from the past, when intravascular ultrasound (IVUS) measurements taught the interventional community that bare metal stents had to be implanted and often postdilated with larger balloons and at higher pressures to improve stent expansion and apposition ${ }^{12,13}$. IVUS use then resulted in an impressive reduction of 
stent thrombosis risk ${ }^{12}$. Careful pre-interventional assessment of reference vessel size and examinations of BVS implanted are likely to improve the clinical, results in BVS too. In most of the recent BVS studies, quantitative coronary angiography was used for BVS sizing, but in some studies IVUS was also performed $^{2}$. Pre-interventional IVUS can be highly valuable for the assessment of true vessel size and both vascular remodelling and tapering ${ }^{14}$. Imaging with OCT can also help to optimise BVS safely and to detect device fractures, which -of course- should be prevented based on pre-interventional measurements of vessel size 11,15 .

Does the current study provide answers to all relevant questions about the overexpansion of bioresorbable scaffolds? Well, probably not. While the authors obtained highly valuable data about Absorb BVS, they did not study other polymer- or magnesium-based bioresorbable scaffolds which have different physical properties ${ }^{16,17}$. For instance, a PLLA-based scaffold, which regains its diameter after acute recoil due to the "self-correcting" properties of the device, was previously shown to have a much greater tolerance to significantly oversized post-dilations than the Absorb BVS ${ }^{16}$. Finally, Foin et al used expired BVS for their experiments, and polymer ageing could have affected the physical properties of the devices tested ${ }^{18}$. Therefore, it would be of great interest to perform additional in vitro studies with fresh samples of the Absorb BVS and other bioresorbable scaffolds, using the same experimental set-up as applied in the current study.

To conclude, well-controlled post-dilatation and overexpansion of current BVS is likely to prevent a substantial proportion of potential scaffold thromboses by improving the BVS apposition to the vessel wall. On the other hand, excessive overexpansion can lead to fractures of the polymer that may induce adverse events such as scaffold thromboses. 3D OCT, a technique which Foin et al used in their current bench-top study to identify BVS fractures, could not only help reveal the mechanisms of thrombus formation in BVS, but has - together with IVUS and other advanced quantitative coronary imaging techniques - the potential to reduce the risk of fracturing struts.

\title{
Funding
}

The Research Department of Thoraxcentrum Twente has received research grants from AstraZeneca, Biotronik, Boston Scientific, and Medtronic.

\section{Conflict of interest statement}

C. von Birgelen has been a consultant for Boston Scientific and Medtronic, and has received speaker's honoraria from MSD and AstraZeneca. The other author has no conflicts of interest to declare.

\author{
S Previous \\ The Mitral Valve Meeting in Zurich = a world upside d...
}

\section{Share this article:}

Facebook Tweet Linkedln Google + Email

\section{AUTHORS}

- von Birgelen C

- Basalus M

\section{REFERENCES}

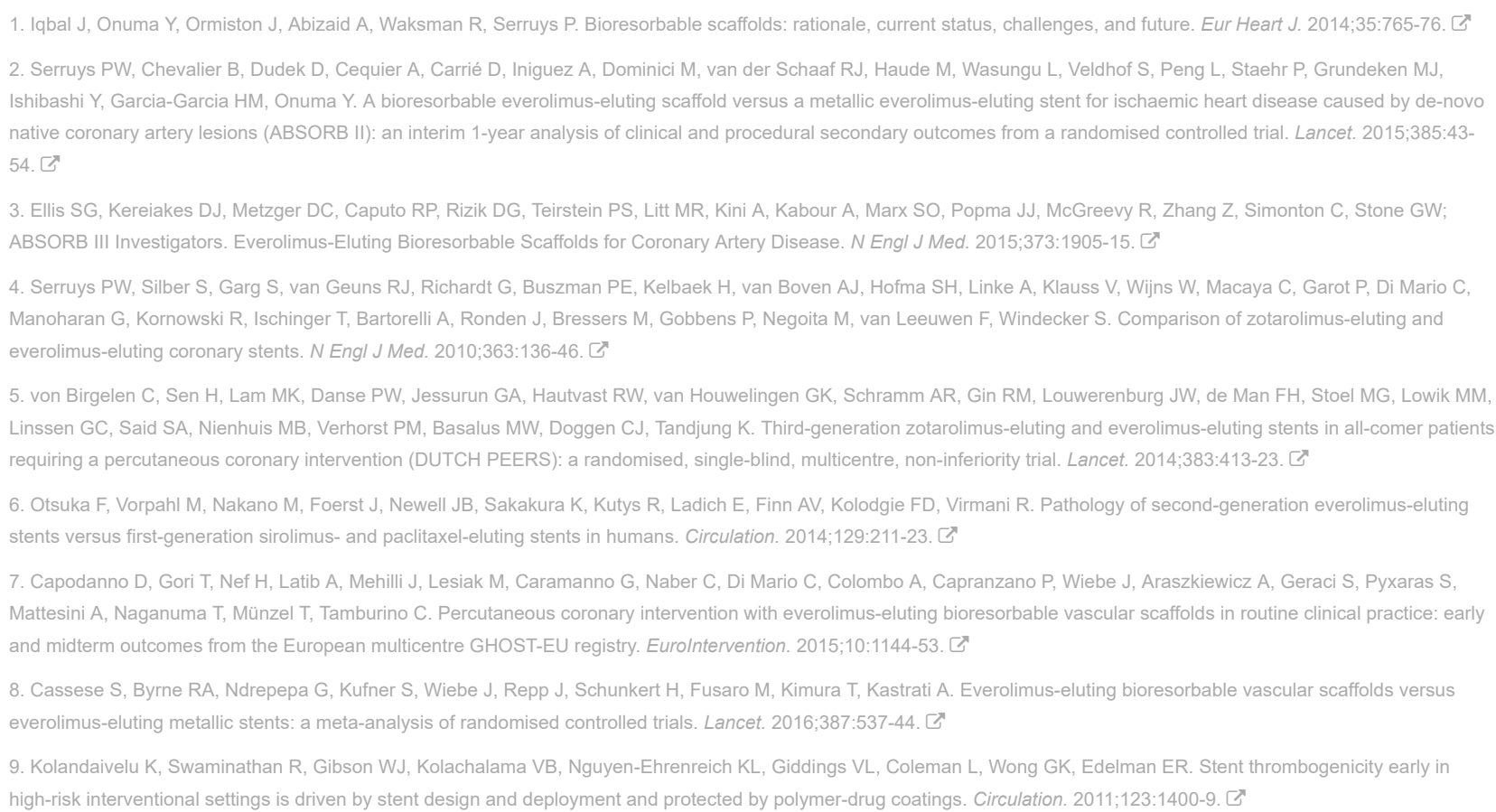


10. Basalus MW, van Houwelingen KG, Ankone MJ, Feijen J, von Birgelen C. Micro-computed tomographic assessment following extremely oversized partial postdilatation of drug-eluting stents. Eurolntervention. 2010;6:141-8. ¿

11. Foin N, Lee R, Mattesini A, Caiazzo G, Fabris E, Kilic D, Chan JN, Huang Y, Venkatraman SS, Di Mario C, Wong P, Nef H. Bioabsorbable vascular scaffold overexpansion: insights from in vitro post-expansion experiments. Eurolntervention. 2016;11:1389-99.

12. Colombo A, Hall P, Nakamura S, Almagor Y, Maiello L, Martini G, Gaglione A, Goldberg SL, Tobis JM. Intracoronary stenting without anticoagulation accomplished with intravascular ultrasound guidance. Circulation. 1995;91:1676-88. ¿

13. von Birgelen C, Gil R, Ruygrok P, Prati F, Di Mario C, van der Giessen WJ, de Feyter PJ, Serruys PW. Optimized expansion of the Wallstent compared with the PalmazSchatz stent: on-line observations with two- and three-dimensional intracoronary ultrasound after angiographic guidance. Am Heart J. 1996;131: 1067-75. ¿

14. von Birgelen C, Mintz GS, de Vrey EA, Kimura T, Popma JJ, Airiian SG, Leon MB, Nobuyoshi M, Serruys PW, de Feyter PJ. Atherosclerotic coronary lesions with inadequate compensatory enlargement have smaller plaque and vessel volumes: observations with three dimensional intravascular ultrasound in vivo. Heart. 1998;79:137-42. ¿

15. Allahwala UK, Cockburn JA, Shaw E, Figtree GA, Hansen PS, Bhindi R. Clinical utility of optical coherence tomography (OCT) in the optimisation of Absorb bioresorbable vascular scaffold deployment during percutaneous coronary intervention. Eurolntervention. 2015;10:1154-9. ¿

16. Ormiston J, Webber B, Ubod B, Darremont O, Webster MW. An independent bench comparison of two bioresorbable drug-eluting coronary scaffolds (Absorb and DESolve) with a durable metallic drug-eluting stent (ML8/Xpedition). Eurolntervention. 2015;11:60-7. C

17. Haude M, Ince H, Abizaid A, Toelg R, Lemos PA, von Birgelen C, Christiansen EH, Wijns W, Neumann FJ, Kaiser C, Eeckhout E, Lim ST, Escaned J, Garcia-Garcia HM, Waksman R. Safety and performance of the second-generation drug-eluting absorbable metal scaffold in patients with de-novo coronary artery lesions (BIOSOLVE-II): 6 month results of a prospective, multicentre, non-randomised, first-in-man trial. Lancet. 2016;387:31-9. ¿

18. Bergström JS, Hayman D. An Overview of Mechanical Properties and Material Modeling of Polylactide (PLA) for Medical Applications. Ann Biomed Eng. 2015 Sep 14. [Epub ahead of print]. [ᄌ 